\title{
Influence of supramolecular forces on the linear viscoelasticity of gluten
}

\author{
Vassilis Kontogiorgos ${ }^{1} \cdot$ Paras Shah $^{2} \cdot$ Paul Bills $^{2}$
}

Received: 30 July 2015 /Revised: 23 September 2015 / Accepted: 13 December 2015 /Published online: 14 January 2016

(C) The Author(s) 2016. This article is published with open access at Springerlink.com

\begin{abstract}
Stress relaxation behavior of hydrated gluten networks was investigated by means of rheometry combined with $\mu$-computed tomography ( $\mu$-CT) imaging. Stress relaxation behavior was followed over a wide temperature range $(0-$ $70{ }^{\circ} \mathrm{C}$ ). Modulation of intermolecular bonds was achieved with urea or ascorbic acid in an effort to elucidate the presiding intermolecular interactions over gluten network relaxation. Master curves of viscoelasticity were constructed, and relaxation spectra were computed revealing three relaxation regimes for all samples. Relaxation commences with a welldefined short-time regime where Rouse-like modes dominate, followed by a power law region displaying continuous relaxation concluding in a terminal zone. In the latter zone, poroelastic relaxation due to water migration in the nanoporous structure of the network also contributes to the stress relief in the material. Hydrogen bonding between adjacent protein chains was identified as the determinant force that influences the relaxation of the networks. Changes in intermolecular interactions also resulted in changes in microstructure of the material that was also linked to the relaxation behavior of the networks.
\end{abstract}

Keywords Gluten · Biopolymer $\cdot$ Relaxation $\cdot \mu$-Computed tomography $\cdot \mathrm{Gel}$

\section{Vassilis Kontogiorgos}

v.kontogiorgos@hud.ac.uk

1 Department of Biological Sciences, School of Applied Sciences, University of Huddersfield, HD1 3DH, Huddersfield, UK

2 Centre for Precision Technologies, School of Computing and Engineering, University of Huddersfield, HD1 3DH, Huddersfield, UK

\section{Introduction}

Gluten networks consist of a complex mixture of wheat storage proteins and are of paramount financial significance as the structure of bakery products and of gluten-based biomaterials depends on their mechanical behavior. On fundamental level, rheological properties are controlled by the interplay of attractive and repulsive supramolecular forces that act between adjacent protein chains. Weak forces typically include hydrogen bonding, hydrophobic interactions, van der Waals forces, $\pi-\pi$ stacking between rings of aromatic amino acids, and ionic interactions. Strong forces (covalent bonding) and in particular disulfide bridges form permanent coupling between sulfur containing amino acids (e.g., cysteine and methionine) (Khan and Shewry 2009). Weak interactions act cooperatively and form labile transient binding partners that can break and reform with a characteristic timescale. Strong forces form crosslinks between protein chains that may also undergo disulfide link interchange especially at high temperatures and with the application of shear (Auvergne et al. 2008). The complexity and multiplicity of interactions that take place at molecular level render to gluten its distinct rheological properties and understanding, and controlling their behavior is a challenging task.

The combination disulfide cross-links with temporally evolving interactions (hydrogen interactions) makes gluten and dough systems good candidates to be described with the Lodge rubberlike model (Lodge 1964). In this model, network junctions can break and reform with a characteristic lifetime thus making the system able to flow. This approach has been used in several instances to describe the mechanical behavior of dough systems (Migliori and Gabriele 2010; Mohammed et al. 2013; Tanner et al. 2007, 2008, 2011). The importance of the molecular interactions on the mechanical behavior of gluten is manifested by the presence of "critical gel" 
characteristics (Gabriele et al. 2001; Migliori and Gabriele 2010; Mohammed et al. 2013; Ng and McKinley 2008; Zuo et al. 2008) as the relaxation modulus follows power law behavior for $\mathrm{t}>\lambda_{o}$ with $\lambda_{o}$ denoting the shortest time below which Rouse-like modes are observed (Winter and Chambon 1986). Thus, gluten is capable of flow although in practice, it is difficult to observe it due to aging effects that change the physicochemical characteristics of the material (e.g., dehydration).

In our previous investigation (Kontogiorgos and Dahunsi 2014), we have shown that compositional and microstructural differences influence the relaxation patterns of gluten composites. In the present work, we build on our previous findings by modulating supramolecular forces (hydrogen bonding and disulfide bridges) that stabilize gluten network with the objective to identify the relative importance of molecular interactions in the relaxation dynamics of gluten.

\section{Materials and methods}

\section{Materials and sample preparation}

Gluten, ascorbic acid, urea, and dithiothreitol (DTT) used in the present investigation were purchased from Sigma-Aldrich (Poole, UK). All samples were prepared at $40 \% w / w$ total protein solids and $60 \% \mathrm{w} / \mathrm{w}$ solvent as described elsewhere (Kontogiorgos et al. 2007). Control sample was hydrated using deionized water. Aqueous solvents were prepared by dissolving the corresponding reagent in deionized water to prepare solutions with final concentrations of 100 or $200 \mathrm{ppm}$ for ascorbic acid, 1 or $4 \mathrm{M}$ for urea, and $2 \% v / v$ for DTT.

\section{Stress relaxation measurements}

Stress relaxation measurements were performed between 0 $70{ }^{\circ} \mathrm{C}$ using a rotational rheometer (Bohlin Gemini 200HRnano, Malvern Instruments, Malvern, UK) equipped with serrated plate-serrated plate geometry $(25 \mathrm{~mm}$ diameter and $1 \mathrm{~mm}$ gap). Shear strain amplitude sweep experiments were performed between $0-70{ }^{\circ} \mathrm{C}$ using angular frequency of $6.28 \mathrm{rad} / \mathrm{s}$ to determine the linear viscoelastic region (LVR) of the samples. Strain (2\%) was found to be within the LVR of all samples and to give satisfactory signal-to-noise ratio. Time sweeps in dynamic oscillation on shear were executed at $6.28 \mathrm{rad} / \mathrm{s}$ and $2 \%$ strain for $60 \mathrm{~min}$ revealing that storage $\left(\mathrm{G}^{\prime}\right)$ and loss moduli $\left(\mathrm{G}^{\prime \prime}\right)$ reach pseudoequilibrium within $15 \mathrm{~min}$. Therefore, samples were left to equilibrate for $15 \mathrm{~min}$ before measurements to dissipate stresses that were created during loading. Stress relaxation tests were carried out using $2 \%$ instantaneous strain for 30 min for each sample. The same specimen was used successively at each temperature (total measurement time $255 \mathrm{~min}$ ). The measurements were performed four times for each sample and averaged curves are reported. Data of stress relaxation modulus $(G(t))$ were collected in logarithmic mode with respect to the timescale of observation. Strain rise time was $20 \mathrm{~ms}$ and data point collection started after $30 \mathrm{~ms}$. A thin layer of low viscosity silicone oil (polydimethylsiloxane, Sigma-Aldrich, St. Louis, MO) was also applied to minimize moisture loss during the course of experiments. Nonlinear regression was performed with GraphPad Prism v.6 (Graph-Pad Software, San Diego, USA).

\section{Numerical computation}

Numerical computation was performed in MATLAB (v7.0 R14 Service Pack 2, The Mathworks Inc., MA) as described previously (Kontogiorgos and Dahunsi 2014). Briefly, the first step involves discretization of stress relaxation function to create matrix $A$ and was performed with the discr.m script published elsewhere (Kontogiorgos et al. 2009). Following that step, algorithms $c s v d . m$ for calculation of the singular value decomposition of matrix $A$ and $l_{-}$curve.m for computation of the optimum regularization parameter were used from Hansen's regularization tool package (Hansen 1994). Finally, the algorithm NLCSmoothReg.m was used for the calculation of the spectra (Wendlandt 2005).

\section{$\mu$-Computed tomography}

Prior to imaging, samples of gluten were fixed in plastic sample holders, sealed, and allowed to relax for a period of $24 \mathrm{~h}$ to minimize structural changes during the course of imaging. Samples were imaged using a Nikon XTH $225 \mu$-computed tomography system (Nikon Metrology, Tring, UK) with a tungsten reflection X-ray target. Datasets were acquired at $150 \mathrm{kV}, 100 \mu \mathrm{A}$ with a resulting voxel size of $6.6 \mu \mathrm{m}$. Each dataset consisted of 1583 individual X-ray projections which were then reconstructed using CT Pro (Nikon Metrology, Tring, UK). Standard algorithms were used for noise reduction and beam hardening compensation. Compensation was carried out using two horizontal slices at opposite extremities of each dataset to take into account any shading gradient across the image. Three-dimensional volume datasets were then produced and post process analysis was performed using VG Studio (Volume Graphics GmbH, Heidelberg, Germany). Data relating to the plastic sample holder and surrounding air was removed from the analysis by demarcation of the dataset shading histogram. An alignment routine was then carried out such that each dataset was aligned correspondingly, and crosssections were then taken at predetermined points through each sample such that qualitative comparison could be performed. 


\section{Results and discussion}

\section{Stress relaxation measurements}

The first step in the investigation was to explore the influence of intermolecular forces on the stress relaxation curve of the specimens. Ascorbic acid acts as oxidizing agent and helps in the formation of additional disulfide bridges with the sulfur containing amino acids of gluten (Joye et al. 2009; Koehler 2003). Conversely, DDT is a strong reducing agent that cleaves disulfide bonds whereas urea exhibits its influence on the hydrogen bonding of the material. All samples were subjected to stress relaxation measurements within the LVR of each sample between $0-70{ }^{\circ} \mathrm{C}$, and data were plotted in double-logarithmic plots (Fig. 1a-d). Temperature increase shows a remarkable influence on the rigidity of the samples with the relaxation modulus decreasing of about one logarithmic cycle between 0 and $70^{\circ} \mathrm{C}$. Control and ascorbic acidtreated samples relax between very similar boundaries $(\log G(t) \sim 3.5-1.8)$ but with distinct relaxation patterns. This is particularly observed beyond $100 \mathrm{~s}(\log t=2)$ where samples treated with ascorbic acid accelerate their relaxation. It should be mentioned that duration of the experiment and temperature might play a role on the chemical reactions induced by ascorbic acid especially at elevated temperatures (e.g., $>30^{\circ} \mathrm{C}$ ). However, development of stress relaxation curves (Fig. 1b) show that any kinetic changes that may occur cannot be rheologically captured by the present experimental protocol. Treating samples with urea (Fig. 1c) brings about an entirely different phenomenology with samples having reduced rigidity and even faster long relaxation processes at all temperatures. Samples treated with DTT did not form a network but a viscous liquid and relaxation modulus relaxes to nearly zero (Fig. 1d), a behavior typical of unlinked macromolecules (Lin 1986). It is evident that in the absence of disulfide bonds, gluten is unable to form a three-dimensional network. Interestingly, by increasing temperature beyond $20^{\circ} \mathrm{C}$, the samples started gelling thus changing the mechanical properties of the material and the experiments had to be terminated. Similar gelling behavior using increasing amounts of $\beta$ mercaptoethanol has been previously observed for gluten likely through the formation of intermolecular linkages of $\beta$-sheet protein structures (Dahesh et al. 2016). Consequently, disulfide cross-links play integral role in gluten network formation and part of the viscoelastic properties should be attributed to their presence, as it will be described below.

Long relaxation events appear to be the distinctive element of the stress relaxation curves of the samples. These delayed processes have been associated with the relaxation of the entire network structure (Kontogiorgos and Dahunsi 2014; Li et al. 2003), and the influence of molecular interactions on network structure is evident particularly at lower temperatures $\left(0-30{ }^{\circ} \mathrm{C}\right)$ and beyond $100 \mathrm{~s}(\log t=2)$. Recent work has shown that the long relaxation events are related to the residual stresses remaining in the structure due to loading of the sample thus creating difficulties in accessing times greater than the rest time that was given to the sample on the rheometer before measurement ( $\mathrm{Ng}$ and McKinley 2008). It is possible by plotting the first derivatives of stress relaxation curves to observe the onset of the long time decays (Fig. 2). Figure 2a
Fig. 1 Double logarithmic plots of stress relaxation curves between $0-70{ }^{\circ} \mathrm{C}$ for a untreated gluten (control), b ascorbic acidtreated gluten, $\mathbf{c}$ urea-treated gluten, and d DTT-treated gluten. Instantaneous strain was $2 \%$ followed by $30 \mathrm{~min}$ relaxation
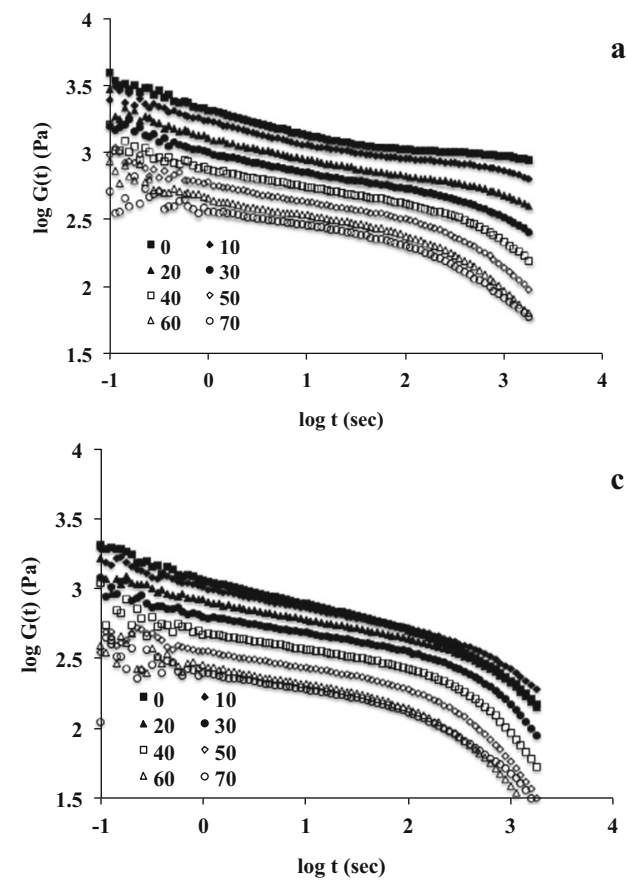

b 

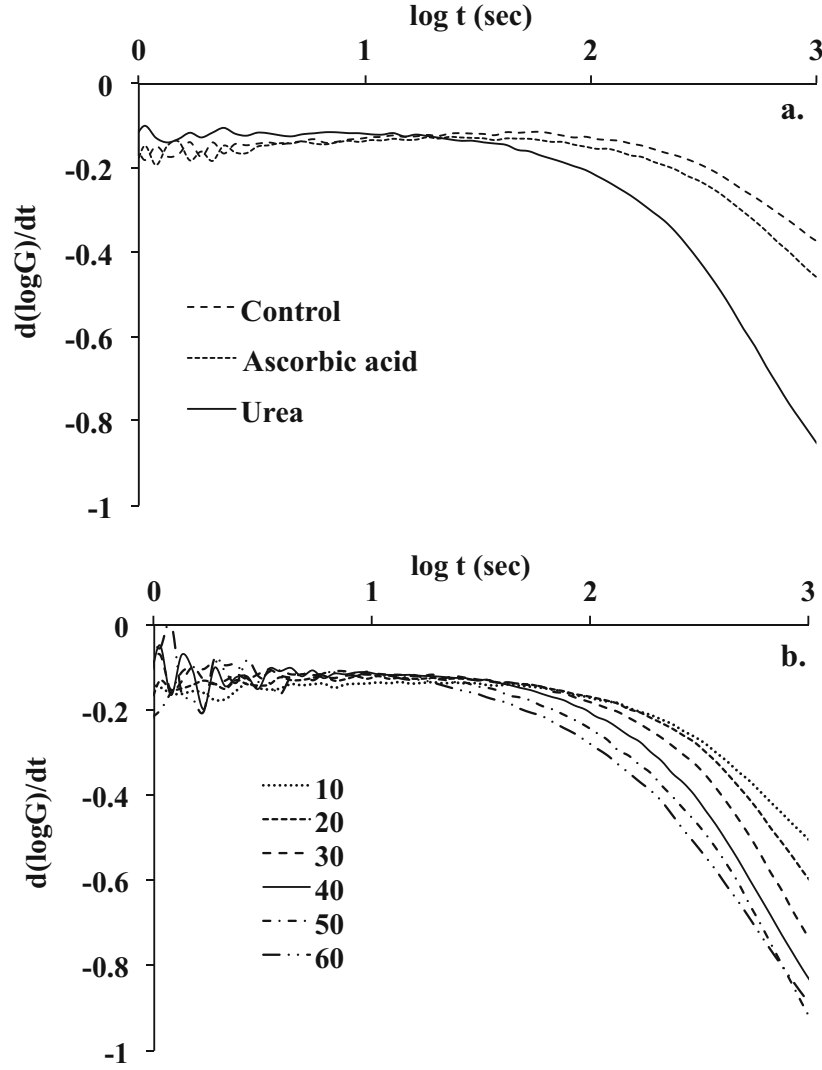

Fig. 2 Typical first derivative of stress relaxation curves showing the influence of a intermolecular forces at $30^{\circ} \mathrm{C}$ and $\mathbf{b}$ temperature on the onset of the terminal relaxation regime for samples treated with urea

shows the influence of intermolecular forces at $30{ }^{\circ} \mathrm{C}$ on the samples. It can be seen that disruption of hydrogen interactions has the greatest influence on the onset of long relaxation processes. Furthermore, temperature increase also accelerates the emergence of the long processes as chain mobility increases with temperature (Fig. 2b). Since all samples were given the same time of rest before measurements $(15 \mathrm{~min})$, the results reveal that temperature as well as the specific structural details of the material also play role on the development of the terminal zone. As a result, the onset of long time relaxation regime is not only a function of rest time but also of temperature and of the molecular interactions in the material.

To further probe relaxation dynamics of gluten network, the stress relaxation curves were treated using the timetemperature superposition principle that enables extending the monitoring of relaxation modulus beyond the experimental timeframe.

\section{Time-temperature superposition}

Time-temperature superposition (TTS) principle has been previously used for gluten (Dahesh et al. 2016; Kontogiorgos and Dahunsi 2014) to investigate relaxation mechanisms at the farthest experimental limits of viscoelasticity. In the present investigation, we used the method of reduced variables to calculate the horizontal shift factors $\left(\mathrm{a}_{\mathrm{T}}=\mathrm{t} / \mathrm{t}_{\mathrm{O}}\right)$ and construct the master curve of viscoelasticity at reference temperature of $\mathrm{T}_{\mathrm{o}}=20^{\circ} \mathrm{C}$. The master curves extended the experimental timeframe of observation for four logarithmic cycles and revealed similar relaxation behavior for all samples irrespectively of the treatment (Fig. 3). Samples treated with urea exhibited a drastic reduction in the values of relaxation modulus. Ascorbic acid-treated networks revealed faster short time relaxations $\left(\log \left(t / a_{T}\right)<-1\right)$; nevertheless, they did not show any appreciable differences with the untreated networks (control) for $\log \left(\mathrm{t} / \mathrm{a}_{\mathrm{T}}\right)>-1$.

The overall reduction of relaxation modulus and the quantitative differences in the short time relaxation regime (log $\left.\left(\mathrm{t} / \mathrm{a}_{\mathrm{T}}\right)<-1\right)$ demonstrate profoundly the pivotal role of hydrogen bonding and disulfide linkages in gluten network structuring and stability. At present, two schools of thought (MacRitchie 2014) have been used to describe gluten structuring and its rheological response: the particulate (Domenek et al. 2004; Don et al. 2003a, 2003b; Hamer et al. 2005; Lefebvre et al. 2003) and the polymeric (Belton 1999; Singh and MacRitchie 2001) network models. Rheological (Kontogiorgos and Dahunsi 2014; Ng and McKinley 2008), microstructural (Jiang et al. 2008), and calorimetric modeling (Kontogiorgos and Goff 2006) demonstrate a closer alignment of hydrated gluten networks to polymeric rather than the particulate view. Additionally, recent work using a combination of scattering techniques in water/ethanol at the dilute and concentrated regime show that gluten proteins behave as flexible polymer in a good solvent (Dahesh et al. 2014). Utilizing, therefore, the polymeric theoretical framework is possible to

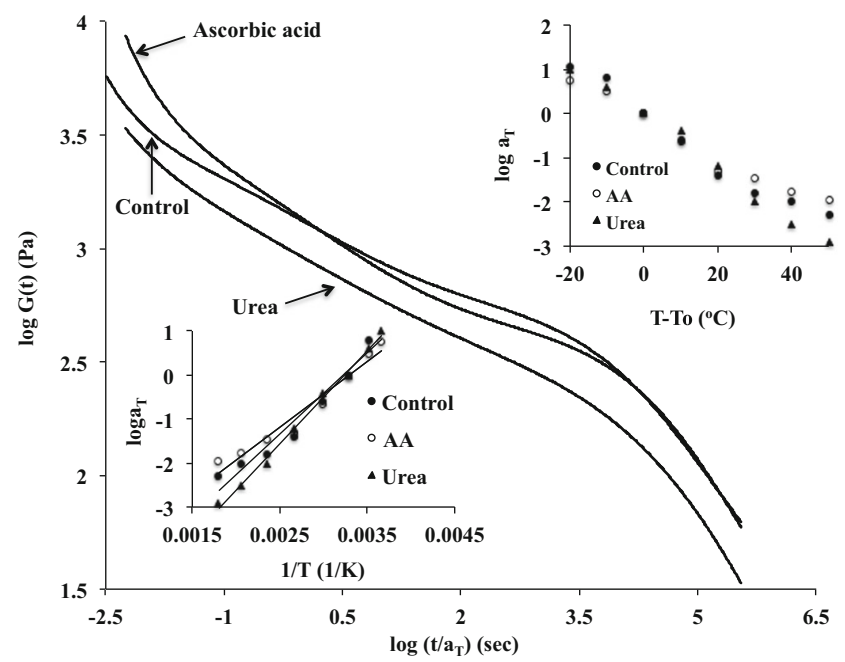

Fig. 3 Double-logarithmic plots of mastercurves of stress relaxation modulus against reduced time at $\mathrm{T}_{\mathrm{o}}=20{ }^{\circ} \mathrm{C}$ for all samples. Top right inset shows the temperature dependence of shift factors $\left(a_{T}\right)$ plotted against $\mathrm{T}_{\mathrm{o}}$. Bottom left inset shows the calculations of the activation energy in semi logarithmic plots of $\log _{\mathrm{T}}$ versus $1 / \mathrm{T}$ 
divide gluten relaxation into three distinct regimes: a short time relaxation process $\left(\mathrm{t} / \mathrm{a}_{\mathrm{T}}<-1\right)$ that corresponds to Rouse-like modes, an intermediate power law relaxation regime $\left(-1<\mathrm{t} / \mathrm{a}_{\mathrm{T}}<-3.5\right)$, and a terminal regime $\left(\mathrm{t} / \mathrm{a}_{\mathrm{T}}>3.5\right)$. It follows that the short time "glassy" modulus $\left(G_{\mathrm{g}}\right)$ is described by:

$$
G_{g}=\frac{k T}{b^{3}}
$$

where $k$ is the Boltzmann's constant, $T$ is the absolute temperature, and $b$ is the length scale that is approximately equal to the size of the segments with Rouse-like relaxation times. From Fig. 3, at $\mathrm{t} / \mathrm{a}_{\mathrm{T}}=-1, \mathrm{G}_{\mathrm{g}}$ is $\sim 2300 \mathrm{~Pa}$ for gluten and ascorbic acid-treated samples and $\sim 1500$ Pa for urea-treated samples. The outcome of the calculations using Eq. 1 yields $b=16 \mathrm{~nm}$ for urea-treated and $14 \mathrm{~nm}$ for the other two samples, values that are in the same range with those previously reported $(b=20 \mathrm{~nm},(\mathrm{Ng}$ and McKinley 2008)). These values are also consistent with nanopore diameter values $(\sim 10$ $14 \mathrm{~nm}$ ) from porosimetric modeling of gluten (Kontogiorgos and Goff 2006) indicating that the mesoporous nature of gluten should be taken into account at these length scales as it will be discussed later. However, from TEM imaging of gluten, gliadin- and glutenin-enriched networks (Jiang et al. 2008) is difficult to discern elementary structures at this length scale as they appear to form continuous sheet-like building blocks. At long times, the equilibrium modulus $\left(\mathrm{G}_{\mathrm{e}}\right)$ is described as:

$$
G_{e}=v k T
$$

with $\nu$ being the number density of network strands. The relaxation patterns of the samples are typical of polydisperse entangled polymeric material with absence of equilibrium modulus. However, by employing the first principles, it is possible to calculate an approximate number density of strands at the point of inflection of the mastercurves at about $\log \left(\mathrm{t} / \mathrm{a}_{\mathrm{T}}\right)=-3.5$ where the final relaxation mechanisms commence. Calculations using Eq. 2 yield $\nu$ values of $8.710^{22} \mathrm{~m}^{-3}$ for control and ascorbic acid-treated, $5.410^{22} \mathrm{~m}^{-3}$ for ureatreated samples. This is an indication that for urea-treated samples, more macromolecules have relaxed due to higher mobility of protein chains. Such a behavior is based on the direct mechanism in which urea cooperates with water in the solvation of amino acid residues and disrupts hydrogen bonding. In this model, the weakening of hydrophobic interactions occurs because urea is more effective than water in solvating hydrophobic residues (Rezus and Bakker 2006) thus accelerating mobility and relaxation processes. In contrast, creation of additional disulfide linkages does not seem to influence the long relaxation processes of the samples.

To further explore the effect of temperature on the relaxation dynamics, the horizontal shift factors $\mathrm{a}_{\mathrm{T}}$ were plotted against T-To (Fig. 3, top right inset). Shift factors did not differ substantially between the samples up to $40{ }^{\circ} \mathrm{C}(\mathrm{T}-\mathrm{To}=20)$ showing similar dependence of relaxation mechanisms on temperature. However, beyond $40{ }^{\circ} \mathrm{C}$, samples treated with urea or ascorbic acid show stronger or weaker dependency on temperature, respectively. Increase in temperature generally weakens hydrogen bonding, and further disruption of interactions with urea results in faster relaxation dynamics and stronger temperature dependency of these samples. Temperature dependence of $\mathrm{a}_{\mathrm{T}}$ can be described with the Arrhenius relationship:

$\log a_{T}=\frac{E_{a}}{R}\left(\frac{1}{T}-\frac{1}{T_{0}}\right)$

where $E_{a}$ is the activation energy for an elementary relaxation and $R$ the gas constant. Calculation of the activation energy can be performed with semi logarithmic plots of $\operatorname{loga}_{\mathrm{T}}$ versus $1 / \mathrm{T}$ (Fig. 3, bottom left inset) yielding 35,28 , and $40 \mathrm{~kJ} / \mathrm{mol}$ for control, ascorbic acid- and urea-treated samples, respectively. Activation energy for an elementary relaxation event seems to be marginally greater for urea-treated samples. Urea disrupts hydrogen bonding and as a consequence, enhances the interaction of hydrophobic amino acids as stated earlier. The strength of hydrophobic interactions usually increases with temperature, and therefore, the system needs to overcome greater energetic barrier thus requiring greater energy for an elementary macromolecular motion. In contrast, ascorbic acid-treated samples require less energy as the additional elastically active disulfide bonds through oxidative cross-linking accelerate relaxation of protein chains.

An approach that allows obtaining further insights into the relaxation processes of gluten supramolecular networks can be achieved with the calculation of the relaxation spectra that is described in the next section.

\section{Continuous relaxation spectra}

Fredholm integrals of the first kind can be used as the generalized function that describes the stress relaxation curve:

$g(s)=\int_{0}^{a} K(s, t) H(t) d t, 0 \leq s \leq a$

where $K(s, t)$ is the exponential kernel $\exp (-t / s)$ describing the decay, $g(s)$ is the measured signal (i.e., $G(t)$ ), and $H(t)$ is the unknown integral solution that represents the continuous relaxation spectrum of the material. The limits of integration are between zero and $\alpha$ that are the beginning and end of the experimental time. Data from mastercurves of viscoelasticity in Fig. 3 were analyzed with Tikhonov regularization to obtain the relaxation spectra of the materials. Discretization of Eq. 4 was performed between the minimum and maximum experimental time points in the mastercurves, and calculation of the optimum regularization parameter, $\lambda_{o p t}$, was estimated using 
the L-curve criterion (Hansen 1994). Figure 4 reproduces double logarithmic plots of such data treatment using $\lambda_{\text {opt }}$. Spectral analysis reveals, as in Fig. 3, the three relaxation zones that can be broadly divided in the "glassy" zone $(\lambda<0.1 \mathrm{~s})$, the transition zone $(0.1<\lambda<1000 \mathrm{~s})$ that exhibits the power-law response, and the terminal zone $\left(\lambda>10^{4} \mathrm{~s}\right)$. The glassy zone represents the elastic response of the protein chains and localized configurational rearrangements of amino acids, side chains, or entanglements. In the transition zone, the modulus will have contributions from hydrogen-bonded protein chains, entangled proteins that do not form disulfide bridges, and from disulfide cross-linked proteins. The modulus in this zone is the product of the number of elastically active network elements and the stored energy per element. In the case of hydrogen-bonded segments of the chains, the sites that store elastic energy break and reform in a time-dependent manner. Disengagement of the chains from the neighboring partner results in release or stress and return of the chain in a lower average energetic state. This is a continuous process that results in the absence of a rubbery plateau due to the polydispersity and complexity of the composition ( $>50$ different protein species). The concept of reversibility of non-covalent transient bonds in network generation is well established in the area of supramolecular polymeric materials. In contrast to "conventional" polymeric materials, the strength of supramolecular systems is generated by the "stickiness" of the transient interactions and not only by the covalent bonds or chain entanglements (Herbst et al. 2013; Seiffert 2015; Seiffert and Sprakel 2012). Sticky reptation dynamics (Bromberg 1998; Leibler et al. 1991; Rubinstein and Dobrynin 1999) is frequently employed in rheology of biological materials, as for instance, in gelatin gels (van Vliet and Walstra 1995), alginate gels (Baumberger and Ronsin 2009), hybrid hydrogels (Sun et al. 2012) or gluten composites (Kontogiorgos and Dahunsi 2014) where supramolecular forces prevail.

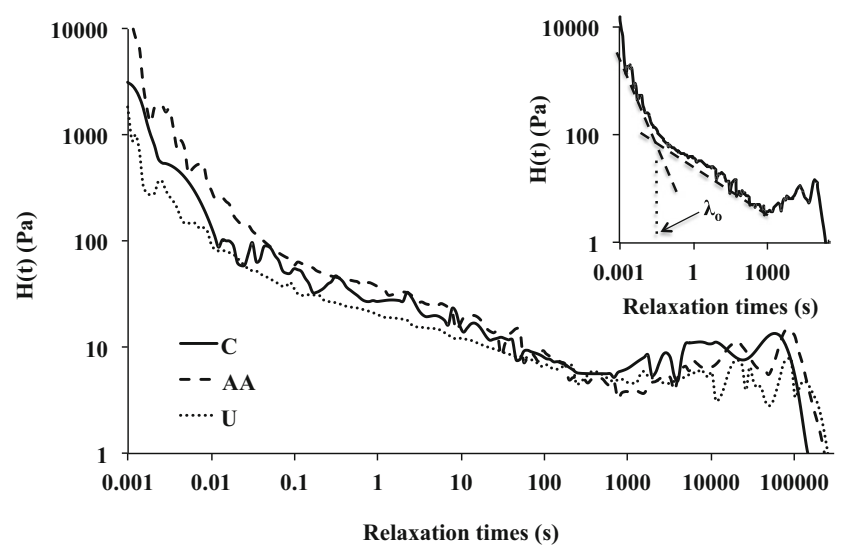

Fig. 4 Double-logarithmic plots of relaxation spectra calculated using data from mastercurves (Fig. 3). Inset shows the way $\lambda_{o}$ was calculated demarcating the transition from Rouse modes to power law relaxation
The behavior in the transition zone is congruent with polymeric gels near the sol-gel transition ( $\mathrm{Ng}$ and McKinley 2008; Winter and Chambon 1986). For critical gels, the relaxation spectrum is given by (Winter and Mours 1997):

$H(\lambda)=\frac{S}{\Gamma(n)} \lambda^{-n}, \lambda_{0}<\lambda<\infty$

where $S$ is the gel strength, $n$ is the power-law exponent, $\lambda_{o}$ the Rouse time (short-time), and $\Gamma(n)$ is the gamma function that is approximated with:

$\Gamma(n)=\frac{1}{n} \Gamma(1+n) \approx \frac{1}{n}\left[1-01138\left(1-4(n-0.5)^{2}\right)\right]$

It can be seen that there is a crossover point from the short times to the power law relaxation regime (Fig. 4, inset). Intersect of tangents of these two regions yields the model parameters of Eq. 5 and the crossover point that corresponds to $\lambda_{o}$ for each sample (Fig. 4, inset and Table 1). Parameters $S$, $n, \lambda_{o}$ depend on the structure of the material (Winter and Mours 1997) and is evident that structural properties are influenced by modulation of intermolecular interactions. In particular, $\lambda_{o}$ is shorter and stiffness $S$ and $n$ are higher for the ascorbic acid-treated samples indicating faster relaxations as a result of additional disulfide linkages in the structure. The terminal zone has contributions from chains that strongly interact with each other and from the relaxation of the entire structure due to interconnectivity. The maximum that is observed in the spectra for all samples is typical of amorphous polymers with high molecular weight (Ferry 1980). This peak indicates that slow relaxation processes have important contribution in the mechanical properties of the samples. In addition to the viscoelastic mechanisms of relaxation, the contribution of solvent migration throughout the nanoporous structure should also be discussed. When an external load is applied in porous systems, the volume fraction of the pores is affected. The fluid-filled pores experience a change in pressure under stress, which leads to fluid motion. In such systems, relaxation is divided into viscoelastic relaxation that has been described already and into poroelastic relaxation due to solvent migration in the pores of the network (Wang et al. 2014; Wang and Hong 2012). This mechanism involves long-range movement of the solvent (aqueous solvent in our

Table 1 Critical gel model parameters for the power-law relaxation regime for all samples

\begin{tabular}{lllll}
\hline Samples & $n$ & $\Gamma(n)$ & $S\left(\operatorname{Pas}^{\mathrm{n}}\right)$ & $\lambda_{o}(\mathrm{~ms})$ \\
\hline Gluten & 0.27 & 3.37 & 104 & 12 \\
Ascorbic acid & 0.35 & 2.91 & 132 & 9 \\
Urea & 0.22 & 4.11 & 83 & 18 \\
\hline
\end{tabular}


samples) and contributes to the terminal relaxation processes (Oyen 2013; Strange et al. 2013; Wang et al. 2014). Poroviscoelastic relaxation analysis has not received attention for gluten networks although some work is available in the literature for other biopolymer systems, as for instance for gelatin (Forte et al. 2015; Galli et al. 2011; Kalyanam et al. 2009), alginate (Cai et al. 2010), or fibrin gels (Noailly et al. 2008).

\section{$\mu$-Computed tomography}

Microstructural observations are integral to rheological measurements as the information that is obtained can be coupled and give insights to the mechanical behavior of the material. Tomography was employed for this purpose, as it is well suited (nondestructive, no sample preparation) to investigate the internal structure of the samples. Figure 5 shows images taken longitudinally from approximately the same plane and region of the sample holder. Beam attenuation as it passes through samples is represented with grayscale voxels that correspond to the density of the microstructure with lighter-colored areas representing denser structures due to the increased beam attenuation (Fig. 5a-c).

Structural features of control samples (Fig. 5a) are qualitatively in good agreement with cryo-SEM (Kontogiorgos and Goff 2006) and confocal laser (Kontogiorgos and Dahunsi 2014) imaging revealing the characteristic features of a perforated porous network. It should be mentioned that these are microscopic pores rather than nanopores that were mentioned in the previous section. Network density seems to be anisotropically distributed throughout the specimen with some areas having lower density than others. Treating samples with ascorbic acid (Fig. 5b) or urea (Fig. 5c) reveals the remarkable influence of intermolecular forces on the microstructure of the material. Samples treated with ascorbic acid (Fig. 5b) display lower beam attenuation whereas in those treated with urea (Fig. 5c), the characteristic topology of gluten network vanishes revealing few distinctive features. Furthermore, defect analysis of the materials indicates the predominance of closepacked micropores (green areas) compared to the control for samples treated with ascorbic acid or urea (Fig. 5d-f). A plausible explanation for the tomographic observations could reside on the modulation of protein-water interactions in the presence of the chemical additives. Gluten proteins exhibit variable strength of interaction with water depending on the proximity of the protein chain to water. In most cases, water molecules in the vicinity of a protein chain exhibit strong interactions (Bosmans et al. 2012). Presence of urea weakens protein-water interactions thus less water is retained in the close proximity of the protein chain resulting in overall greater beam attenuation, whereas oxidative cross-linking in the presence of ascorbic acid exhibits the opposite effect. It is evident that viscoelastic relaxations that were described in the previous sections are linked to the microstructure of the material. For instance, variations in the microporosity (green color, Fig. 5d-f) may also contribute to the differences in the relaxation processes that were observed in the previous sections as protein chains have greater topological freedom to relax in the presence of voids. It should be mentioned that in our previous work (Kontogiorgos and Dahunsi 2014), increasing amounts of gliadin in the network also affected the relaxation behavior and microstructure of the samples in a way similar to urea treatment that could indicate that glutenins are more involved in hydrogen bonding than gliadins are. At this stage, it is rather difficult to quantify the influence of defects in the material but is evident that can be modified by influencing the forces that
Fig. 5 First row shows $\mu$ computed tomographic scanning of samples; from left to right a control, b ascorbic acid-treated, and $\mathbf{c}$ urea-treated gluten. Second row shows defect analysis; from left to right $\mathbf{d}$ control, $\mathbf{e}$ ascorbic acid-treated, and $\mathbf{f}$ urea-treated gluten. Colored scale bar shows defect volume in cubic millimeters. Red $0.000010 \mathrm{~mm}^{3}$, green $0.000005 \mathrm{~mm}^{3}$, and blue $0.000001 \mathrm{~mm}^{3}$
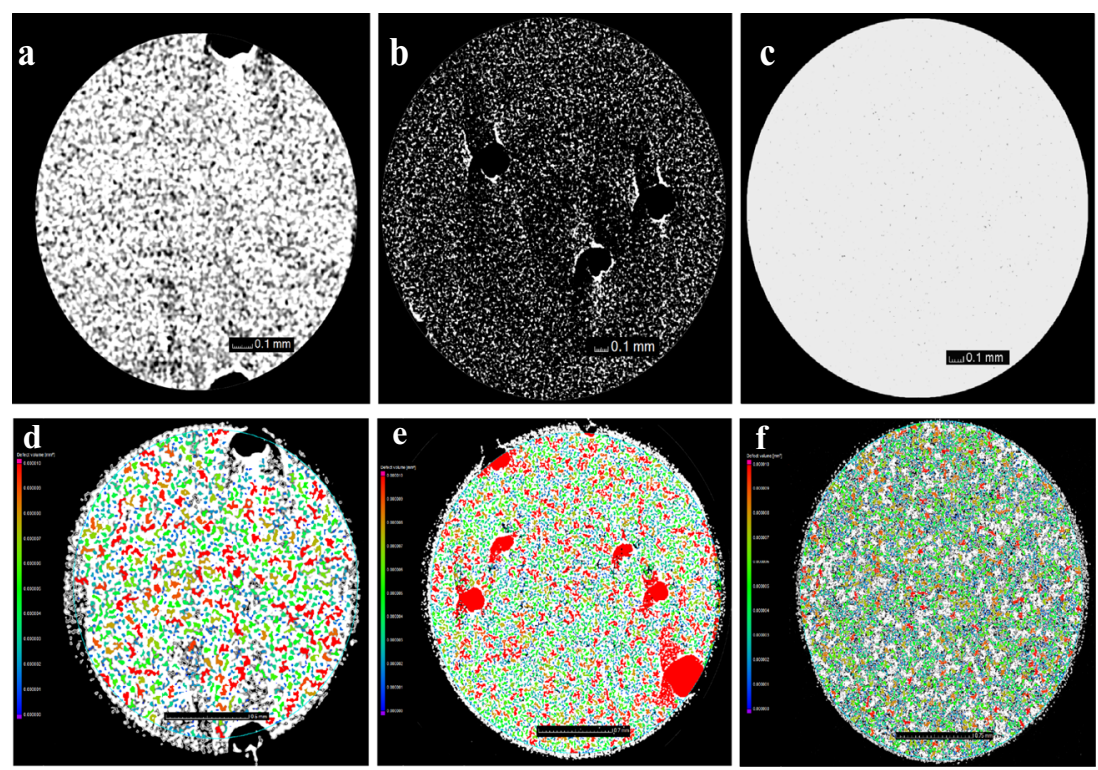
are responsible for structure formation and stabilization. Overall, by controlling the interplay between protein composition and supramolecular interactions, it is possible to tailor the mechanical properties of gluten networks towards creating novel gluten-based biomaterials. It should be stressed that the influence of hydration levels is expected to play significant role, and it remains to be explored.

\section{Conclusions}

Relaxation dynamics of hydrated gluten networks as affected by intermolecular interactions were investigated. Stress relaxation experiments over a broad temperature range and utilization of time-temperature superposition principle augmented the timeframe of experimental monitoring of viscoelastic functions. Hydrogen bonding plays critical role on mechanical relaxations of gluten whereas disulfide bridges seem to influence mostly the Rouse-like modes of relaxation. Spectral analysis of master curves of viscoelasticity revealed that gluten network exhibits power-law behavior at intermediate relaxation times that is indistinguishable from polymeric gels near the sol-gel transition. At long times, gluten displays a dominant relaxation event that apart from the protein chain dynamics is also attributed to poroelastic relaxation due to migration of water in the nanoporous structure. Supramolecular force modulation was also found to affect microstructure and to be coupled to the relaxation characteristics of the networks.

Open Access This article is distributed under the terms of the Creative Commons Attribution 4.0 International License (http:// creativecommons.org/licenses/by/4.0/), which permits unrestricted use, distribution, and reproduction in any medium, provided you give appropriate credit to the original author(s) and the source, provide a link to the Creative Commons license, and indicate if changes were made.

\section{References}

Auvergne R, Morel M-H, Menut P, Giani O, Guilbert S, Robin J-J (2008) Reactivity of wheat gluten protein during mechanical mixing: radical and nucleophilic reactions for the addition of molecules on sulfur. Biomacromolecules 9:664-671. doi:10.1021/bm7009834

Baumberger T, Ronsin O (2009) From thermally activated to viscosity controlled fracture of biopolymer hydrogels. J Chem Phys 130: 061102. doi:10.1063/1.3078267

Belton PS (1999) On the elasticity of wheat gluten. J Cereal Sci 29:103107

Bosmans GM, Lagrain B, Deleu LJ, Fierens E, Hills BP, Delcour JA (2012) Assignments of proton populations in dough and bread using NMR relaxometry of starch, gluten, and flour model systems. J Agr Food Chem 60:5461-5470

Bromberg L (1998) Scaling of rheological properties of hydrogels from associating polymers. Macromolecules 31:6148-6156. doi:10.1021/ ma980523f
Cai S, Hu Y, Zhao X, Suo Z (2010) Poroelasticity of a covalently crosslinked alginate hydrogel under compression. J App Phys 108. doi:10.1063/1.3517146

Dahesh M, Banc A, Duri A, Morel M-H, Ramos L (2014) Polymeric assembly of gluten proteins in an aqueous ethanol solvent. J Phys Chem B 118:11065-11076. doi:10.1021/jp5047134

Dahesh M, Banc A, Duri A, Morel M-H, Ramos L (2016) Spontaneous gelation of wheat gluten proteins in a food grade solvent. Food Hydrocolloids 52:1-10. doi:10.1016/j.foodhyd.2015.06.014

Domenek S, Brendel L, Morel M-HLN, Sp G (2004) Swelling behavior and structural characteristics of wheat gluten polypeptide films. Biomacromolecules 5:1002-1008. doi:10.1021/bm034499b

Don C, Lichtendonk W, Plijter JJ, Hamer RJ (2003a) Glutenin macropolymer: a gel formed by glutenin particles. J Cereal Sci 37: $1-7$

Don C, Lichtendonk WJ, Plijter JJ, Hamer RJ (2003b) Understanding the link between GMP and dough: from glutenin particles in flour towards developed dough. J Cereal Sci 38:157-165. doi:10.1016/ S0733-5210(03)00017-1

Ferry JD (1980) Viscoelastic properties of polymers, 3rd edn. Wiley, New York

Forte AE, D'amico F, Charalambides MN, Dini D, Williams JG (2015) Modelling and experimental characterisation of the rate dependent fracture properties of gelatine gels. Food Hydrocoll 46:180-190. doi:10.1016/j.foodhyd.2014.12.028

Gabriele D, de Cindio B, D'antona P (2001) A weak gel model for foods. Rheol Acta 40:120-127. doi:10.1007/s003970000139

Galli M, Fornasiere E, Jl C, Oyen ML (2011) Poroviscoelastic characterization of particle-reinforced gelatin gels using indentation and homogenization. J Mech Behav Biomed Mater 4:610-617. doi:10. 1016/j.jmbbm.2011.01.009

Hamer RJ, van Vliet T, Lefebvre J (2005) Letter to the editor. J Cereal Sci 42:344-345

Hansen P-C (1994) Regularization tools: a MATLAB package for analysis and solution of discrete ill-posed problems. Numer Algorithm $6: 1-35$

Herbst F, Döhler D, Michael P, Binder WH (2013) Self-healing polymers via supramolecular forces Macromolecular. Rapid Commun 34: 203-220. doi:10.1002/marc.201200675

Jiang B, Kontogiorgos V, Kasapis S, Goff HD (2008) Rheological investigation and molecular architecture of highly hydrated gluten networks at subzero temperatures. J Food Eng 89:42-48

Joye IJ, Lagrain B, Delcour JA (2009) Use of chemical redox agents and exogenous enzymes to modify the protein network during breadmaking - a review. J Cereal Sci 50:11-21. doi:10.1016/j.jcs. 2009.04.001

Kalyanam S, Yapp RD, Insana MF (2009) Poro-viscoelastic behavior of gelatin hydrogels under compression - implications for bioelasticity imaging. J Biomech Eng 131:081005-081005. doi:10.1115/1. 3127250

Khan K, Shewry PR (2009) Wheat: chemistry and technology. AACC, St. Paul, Minnesota

Koehler P (2003) Effect of ascorbic acid in dough:, Ââ reaction of oxidized glutathione with reactive thiol groups of wheat glutelin. J Agric Food Chem 51:4954-4959. doi:10.1021/jf026061t

Kontogiorgos V, Dahunsi OS (2014) Relaxation dynamics in hydrated gluten networks. J Cereal Sci 59:101-108. doi:10.1016/j.jcs.2013. 11.007

Kontogiorgos V, Goff HD (2006) Calorimetric and microstructural investigation of frozen hydrated gluten. Food Biophysics 1:202-215

Kontogiorgos V, Goff HD, Kasapis S (2007) Effect of aging and ice structuring proteins on the morphology of frozen hydrated gluten networks. Biomacromolecules 8:1293-1299

Kontogiorgos V, Jiang B, Kasapis S (2009) Numerical computation of relaxation spectra from mechanical measurements in biopolymers. Food Res Int 42:130-136 
Lefebvre J, Pruska-Kedzior A, Kedzior Z, Lavenant L (2003) A phenomenological analysis of wheat gluten viscoelastic response in retardation and in dynamic experiments over a large time scale. J Cereal Sci 38:257-267

Leibler L, Rubinstein M, Colby RH (1991) Dynamics of reversible networks. Macromolecules 24:4701-4707. doi:10.1021/ma00016a034

Li W, Dobraszczyk BJ, Schofield JD (2003) Stress relaxation behavior of wheat dough, gluten and gluten protein fractions. Cereal Chem 80 : 333-338

Lin YH (1986) Comparison of experiment and the proposed general linear viscoelastic theory. 2. Stress Relaxation line Shape Analysis Macromolecules 19:159-168

Lodge A (1964) Elastic liquids. Academic, New York

MacRitchie F (2014) Theories of glutenin/dough systems. J Cereal Sci 60:4-6. doi:10.1016/j.jcs.2014.02.010

Migliori M, Gabriele D (2010) Effect of pentosan addition on dough rheological properties. Food Res Int 43:2315-2320. doi:10.1016/j. foodres.2010.08.008

Mohammed MAP, Tarleton E, Charalambides MN, Williams JG (2013) Mechanical characterization and micromechanical modeling of bread dough. J Rheol 57:249-272. doi:10.1122/1.4768463

Ng TSK, McKinley GH (2008) Power law gels at finite strains: The nonlinear rheology of gluten gels. J Rheol 52:417-449

Noailly J, Van Oosterwyck H, Wilson W, Quinn TM, Ito K (2008) A poroviscoelastic description of fibrin gels. J Biomech 41:32653269. doi:10.1016/j.jbiomech.2008.09.002

Oyen ML (2013) Mechanical characterisation of hydrogel materials. Int Mater Rev 59:44-59. doi:10.1179/1743280413y.0000000022

Rezus YLA, Bakker HJ (2006) Effect of urea on the structural dynamics of water. Proc Natl Acad Sci U S A 103:18417-18420. doi:10.1073/ pnas. 0606538103

Rubinstein M, Dobrynin AV (1999) Associations leading to formation of reversible networks and gels. Curr Opin Colloid Interface Sci 4:8387. doi:10.1016/S1359-0294(99)00013-8

Seiffert S (2015) Effect and evolution of nanostructural complexity in sensitive polymer gels. Macromol Chem Phys 216:9-22. doi:10. 1002/macp.201400410
Seiffert S, Sprakel J (2012) Physical chemistry of supramolecular polymer networks. Chem Soc Rev 41:909-930. doi:10.1039/c1cs15191f

Singh H, MacRitchie F (2001) Application of polymer science to properties of gluten. J Cereal Sci 33:231-243

Strange DGT, Fletcher TL, Tonsomboon K, Brawn H, Zhao X, Oyen ML (2013) Separating poroviscoelastic deformation mechanisms in hydrogels App Phys Lett 102:031913-031914

Sun J-Y et al. (2012) Highly stretchable and tough hydrogels. Nature 489: 133-136 doi:http://www.nature.com/nature/journal/v489/n7414/ abs/nature11409.html\#supplementary-information

Tanner RI, Dai S-C, Qi F (2007) Bread dough rheology and recoil: 2. Recoil and relaxation. J Non-Newton Fluid 143:107-119. doi:10. 1016/j.jnnfm.2007.02.001

Tanner RI, Qi F, Dai S-C (2008) Bread dough rheology and recoil: I. Rheology J Non-Newton Fluid 148:33-40. doi:10.1016/j.jnnfm. 2007.04.006

Tanner RI, Qi F, Dai S (2011) Bread dough rheology: an improved damage function model. Rheol Acta 50:75-86. doi:10.1007/s00397010-0512-3

van Vliet T, Walstra P (1995) Large Deformation and fracture behaviour of gels Faraday. Discuss 101:359-370. doi:10.1039/fd9950100359

Wang X, Hong W (2012) A visco-poroelastic theory for polymeric gels. P R Soc A 468:3824-3841. doi:10.1098/rspa.2012.0385

Wang Q-M, Mohan A, Oyen M, Zhao X-H (2014) Separating viscoelasticity and poroelasticity of gels with different length and time scales. Acta Mech Sin 30:20-27. doi:10.1007/ s10409-014-0015-z

Wendlandt M (2005) NLCSmoothReg. http://www.mathworks.com/ matlabcentral/fileexchange/7712.

Winter HH, Chambon F (1986) Analysis of linear viscoelasticity of a crosslinking polymer at the gel point. J Rheol 30:367-382

Winter HH, Mours M (1997) Rheology of polymers near liquid-solid transitions. In: Neutron spin echo spectroscopy viscoelasticity rheology, vol 134. Advances in Polymer Science. Springer, Berlin Heidelberg, pp. 165-234. doi:10.1007/3-540-68449-2_3

Zuo M, Song Y, Zheng Q (2008) Influence of equibiaxial extensional strain on stress relaxation of glycerol plasticised wheat gluten. J Sci Food Agric 88:2350-2356. doi:10.1002/jsfa.3356 